Determination of free fatty acids in plasma under diverse conditions

1. Following an intravenous injection of $250 \mu \mathrm{g}$ adrenaline over a period of $2 \mathrm{~min}$., a steep increase in the free fatty acid content of both arterial and venous blood was observed within $10 \mathrm{~min}$. The arteriovenous difference, which before the injection of adrenaline is very small, increased to a maximum after $10 \mathrm{~min}$., and returned to the original value after $20 \mathrm{~min}$. The arterial blood was sampled from the $A$. bracbialis dextra, and the venous sample was obtained by inserting an approximately $20 \mathrm{~cm}$ long catheter deeply into the muscle tissue of the left forearm (Fig. 6).

2. Arterio-coronary-venous difference in patients suffering from cardiac insufficiency were subjected to extreme strain on a bicycle ergometer. As already reported for athletes who go in for top-strain sports $(8,9)$, the con- centration of free fatty acids in their blood is seen to increase during the physical exertions. The concentration of free fatty acids is at its greatest after 3 min. of maximum exertion. Obviously, the arterio-coronary-venous difference during maximum exertion becomes greater as a result of an increasing extraction of free fatty acids by the heart muscle (Fig. 7).

3. The determination of free fatty acids in the plasma of horses in rest and after a training of $20 \mathrm{~min}$. was made and the results are given in table 4 .

Tab. 4

Free fatty acids ( $\mu$ equiv. $/ \mathrm{ml}$ ) in the plasma of horses in rest and after training of $20 \mathrm{~min}$.

\begin{tabular}{llc}
\hline horses & rest & after exercise \\
\hline P. & 0.048 & 0.178 \\
D. & 0.117 & 0.176 \\
P. W. & 0.078 & 0.261 \\
R. & 0.048 & 0.245 \\
\hline
\end{tabular}

\title{
References
}

1. Dole, V. P., J. Clin. Invest. 35, 150 (1956). - 2. Gordon, R. S. jr., and A. Cherkes, J. Clin. Invest. 36, 810 (1957). - 3. Nelson, G. H., Amer. J. Obstetr. Gynec. 92, 202 (1965). 4. Kelley, T. F., Analytic. Chem. 37, 1078 (1965). - 5. Dole, V. P. and H. Meinertz, J. biol. Chemistry 235, 2595 (1960). 6. Trout, D. L., E. H. Estes, jr., and S. J. Friedberg, J. Lipid Res. 1, 199 (1960). - 7. LinNer, N., Automatic titration of free fatty, acids in plasma, ST 48, Radiometer, Copenhagen, Denmark. - 8. Fredrickson, D. S. and R. S. Gordon, Physiol. Rev. 38 , 585 (1958). - 9. Keul, J., E. Doll, H. Steim, H. Homburger, H. Kern and H. ReIndell, Pflügers Arch. Physiol. 282, 1 (1965). - 10. Keul, J., E. Dold, H. Sterm, H. Homburger, H. KerN and H. ReINDELL, Zsschr. Kreisl.forsch. 55, 190 (1966).

Doz. Dr. J. Keul

Medizinische Univ. Klinik 78 Freiburg

Hugstetterstr. 55

\section{Isolierung eines Xylose-haltigen Glykoproteids aus dem Erythrocyten-Stroma ${ }^{1}$ )}

\author{
Von H. WeICKER \\ Aus der Abteilung für Stoffwechselforschung (Leiter: Prof. Dr. H. Weicker) der Medizinischen Universitäts-Poliklinik \\ Heidelberg (Direktor: Prof. Dr. H. Plügge)
}

(Eingegangen am 27. März 1968)

\begin{abstract}
Aus dem Erythrocyten-Stroma von 25 Blutspenderkonserven, die nach den Blutgruppeneigenschäften $\mathrm{A}, \mathrm{B}, 0, \mathrm{MN}, \mathrm{Rh}$ und nach der Länge der Lagerungszeit der Konserven klassifiziert worden waren, wurden mit dem Phenol-Wasser-System nach der Methode von Westphal und Mitarbeitern, modifiziert von UHLENBRUCK und Mitarbeitern, zwei chemisch und serologisch unterschiedliche Glykoproteide dargestellt.

Das Glykoproteid (W), isoliert aus der Wasser-Phase, enthält MN-Antigen und Virus-Rezeptoren. Wir konnten im wesentlichen die in der Literatur beschriebenen chemischen Eigenschaften dieser Substanz bestätigen, fanden jedoch, daß die Ausbeute von den Blutgruppeneigenschaften und der Lagerungszeit der Konserven abhängig war. Bei 0- und AB-Erythrocyten war die Ausbeute am höchsten und zeigte auch die geringste Beeinflussung durch längere Lagerungszeit. Entgegen den Befunden anderer Autoren fanden wir bei den $M$-Erythrocyten den höchsten Neuraminsäuregehalt, der bei $22-24 \%$ lag und zu $85-90 \%$ enzymatisch abspaltbar war. Die Kohlenhydrat-Komponenten des Glykoproteids (W) waren Galaktose, Mannose, Fucose in einer Verteilung von 4:2:1 sowie Galaktosamin, Glucosamin in einer Verteilung von 5:3. Der Gesamtkohlenhydratgehalt betrug 37-41\%. Insgesamt waren 16 Aminosäuren chromatographisch zu identifizieren.

Das bis jetzt noch nicht beschriebene Glykoproteid (P) wurde aus der Phenol-Phase nach Entfernung der Lipide isoliert und enthielt Xylose in einer Konzentration von 3-3,5\%. Es hatte einen geringeren Neuraminsäuregehalt (8-10\%) als das Glykoproteid (W). Die Neuraminsäure war nur zu 60-70\% abspaltbar. Seine Kohlenhydrat-Komponenten waren Galaktose, Mannose, Fucose, Xylose, Glucose in einer Verteilung von 4:2:1:3:1 und Galaktosamin, Glucosamin in einer Verteilung von 3:2 bei einem Gesamtkohlenhydratgehalt von $20-25 \%$. Die Aminosäure-Zusammensetzung zeigte keine wesentlichen Unterschiede zu dem Glykoproteid (W). Das Glykoproteid (P) besaß keine $\mathrm{MN}$-Antigen-Eigenschaften und hemmte die Virus-Hämagglutination nur geringfügig.
\end{abstract}

1) Mit Unterstützung der Deutschen Forschungsgemeinschaft. 


\section{The isolation of a xylose-containing glycoprotein from erytbrocjte stroma}

Wc isolated tivo glycoproteins from the stroma of erythrocytes using the phenol-water-system of WeSTPFAL and coworkers, modified by UHLENBRUCK and coworkers.

The glycoprotcin (W) with $\mathrm{MN}$ antigens and virus receptor properties was derived from the water phase. It has already been characterised by many investigators.

The second, xylose-containing glycoprotein $(P)$, described here for the first time, was isolated from the phenol phase after the lipids and glycolipids had been removed by extraction with organic solvents. The investigations were carried out with the stroma of erythrocytes derived from 25 different blood donors. Each stroma preparation was specified according to the A, B, $0, M N$ and $R h$ blood group properties, and the different storage time of the blood.

We were able to confirm in general the chemical and serological properties of glycoprotein (W) containing MN antigens and virus receptors described by other authors. However, we found in addition that the yield depended strongly in the ABO blood group properties and the storage time of the blood. The highest yield was found with $\mathrm{AB}$ and 0 erythrocytes, which were influenced less by the storage time than $A$ and $B$ erythrocytes. The glycoprotein with $M$ properties had the highest content of sialic acid (22-24\%), of which up to $85-90 \%$ could be liberated by neuraminidase. The carbohydrate components are galactose, mannose and fucose in a ratio of $4: 2: 1$, and galactosamine and glucosamine in a ratio of $5: 3$. The total amount of carbohydrates was $37-41 \%$. 16 amino acids were identified chromatographically.

The second glycoprotein $(\mathrm{P})$, isolated from the phenol phase after the lipids and glycolipids had been removed, contains $3-3.5 \%$ xylose and has no MN antigens and less virus receptor proterties. The sialic acid content ( $8-10 \%)$ is lower than that of the glycyprotein (W) The sialic acid can be liberated enzymically only up to $60-70 \%$. The carbohydrate components are galactose, mannose, fucose, xylose and glucose in a ratio of $4: 2: 1: 3: 1$. The galactosamine: glucosamine ratio is $3: 2$. The total carbohydrate amount was $20-25 \%$.

Die Glykoproteide des Erythrocyten-Stromas sind für die physiko-chemischen Eigenschaften und die Rezeptor-Funktionen der Erythrocyten-Oberfläche von großer Bedeutung. Als Träger der MN-Blutgruppen-Antigene und der Virus-Rezeptoren sind diese Stroma-Glykoproteide durch eingehende serologische und chemische Untersuchungen recht gut charakterisiert (1-24). Zur Isolierung des Glykoproteids aus dem Stroma hat sich die von WestphaL und Mitarbeitern (25) ausgearbeitete und von UHLENBRUCK und SCHMird (15) modifizierte Darstellungsmethode mit einem Phenol-Wasser- $\mathrm{NaCl}$ System gut bewährt. Bei dem Vergleich der mitgeteilten Untersuchungsergebnisse fällt jedoch auf, daß bei der schon theoretisch zu erwartenden geringen Glykoproteid-Ausbeute die Schwankungsbreite des Gesamtkohlenhydratgehaltes und der prozentualen Verteilung der einzelnen Kohlenhydratkomponenten des Glykoproteids relativ groß ist $(2,3,4,12,15,19,20)$. Dies dürfte in erster Linie darauf zurückzuführen sein, daß bei der kleinen Glykoproteid-Ausbeute weitere Reinigungsverfahren nur bedingt möglich sind und zur Durchführung von Einzelanalysen nur geringe Einsatzmengen zur Verfügung stehen.

'Von den meisten Untersuchern wurde deshalb die Isolierung des Glykoproteids aus gepooltem Spenderblut ohne Klassifizierung der Einzelproben nach verschiedenen Blutgruppen-Systemen vorgenommen. Die Ergebnisse wurden vorwiegend bei 0-Erythrocyten gewonnen, da hierbei die höchste Glykoproteid-Ausbeute zu erzielen war. Weiterhin wurde in der Aufarbeitung der Erythrocyten weder die Lagerungszeit der Blutkonserven berücksichtigt, noch wurden Vergleichsuntersụchungen zwischen dem Neuraminsäuregehalt des isolierten Glykoproteids und dem Neuraminsäuregẹhalt der Nativ-Erythroçyten durchgeführt.

Unter Berücksichtigung dieser Gesichtspunkte klassifizierten wir unser Untersuchungsmaterial sowohl nach AB0-, MN- und Rh-Eigenschiaften als auch nach der unterschiedlichen Lagerungszeit der Blutkonserven. Das isolierte Stroma-Glykoproteid wurde durch Ionenaus-
tauscher-Chromatographie einer weiteren Reinigung unterzogen. Nach Modifikation einzelner AnalysenMethoden war es möglich, die chemische und serologische Charakterisierung der Substanz bei einer Gesamtmenge von 16-18 mg mit gut reproduzierbaren Werten vorzunehmen.

Da wir außer dem Glykoproteid (W) mit MN-Antigenund Virus-Rezeptor-Eigenschaften noch ein Xylosehaltiges Glykoproteid (P) ohne diese serologischen Merkmale erstmalig in dem Stroma nachweisen konnten, war die Gegenüberstellung dieser beiden Glykoproteide recht aufschlußreich.

\section{Material und Methoden}

Die Glykoproteid-Isolierung wurde aus 25 Blutspenderkonserven von 450 bis $500 \mathrm{~m} /$ Blut vorgenommen, die neben den Blutgruppeneigenschaften $\mathrm{MN}, \mathrm{Rh}, \mathrm{AB} 0$ auch nach der Lagerungszeit der Konserven in Gruppen von 1-5 Tagen, 5-20 Tagen und älter als 20 Tagen eingeteilt worden waren. Im Einzel-Untersuchungsansatz wurden 220 bis $250 \mathrm{ml}$ gewaschene sedimentierte Erythrocyten verwandt.

Das $M N$-Antigen-baltige Glykeoproteid (W) wurde aus der NaCl-haltigen wäßr. Phase nach Westphal und Mitarbeitern (25), modifiziert nach UHLENBRUCK und Schmid (15) isoliert.

Die Darstellung eines bis jetzt noch nicht besclbriebenen Glykoproteids $(P)$ der Erythrocyten-Membran wurde wie folgt vorgenommen: Nach 2-tägiger Dialyse der Phenolphase gegen fließendes Wasser wurde 2 Tage gegen dest. Wasser dialysiert. Der Dialyserückstand wurde gefriergetrocknet und nach Pulverisierung des Lyophilisates mit Methanol und anschließend mit Chloroform: Methanol = 2:1 ( $v / v)$ am Rückfluß jeweils 8 Stdn. bei $50-60^{\circ}$ extrahiert. Nach Extraktion der Lipide wurde der trockene Rückstand in Wasser suspendiert und nach mehrfachem Zentrifugieren und wiederholtem Aufschwemmen des Zentrifugates der wäßr. Uberstand, in dem sich das Xylose-haltige Glykoproteid befand, dialysiert und gefriergetrocknet (Abb. 1).

\section{Reinigung der Glykeoproteide}

Die Glykoproteide (W) und (P) des Stromas wurden 1 proz. in Wasser gelöst und die wasserunlöslichen Verunreinigungen durch Zentrifugation bei 17000 U./Min. abgetrennt. Der Uberstand wurde nach .Dialyse gefriergetrocknet. Das Lyophilisat wurde in wäßr. Lösung über Sephadex G 150 gelfiltriert oder an einer Ionenaustauschersäule Amberlite CG $50 \mathrm{II}, \mathrm{H}^{+}$-Form 100-200 mesh, chromatographiert. Die Verwendung des Ionenaustauschers 


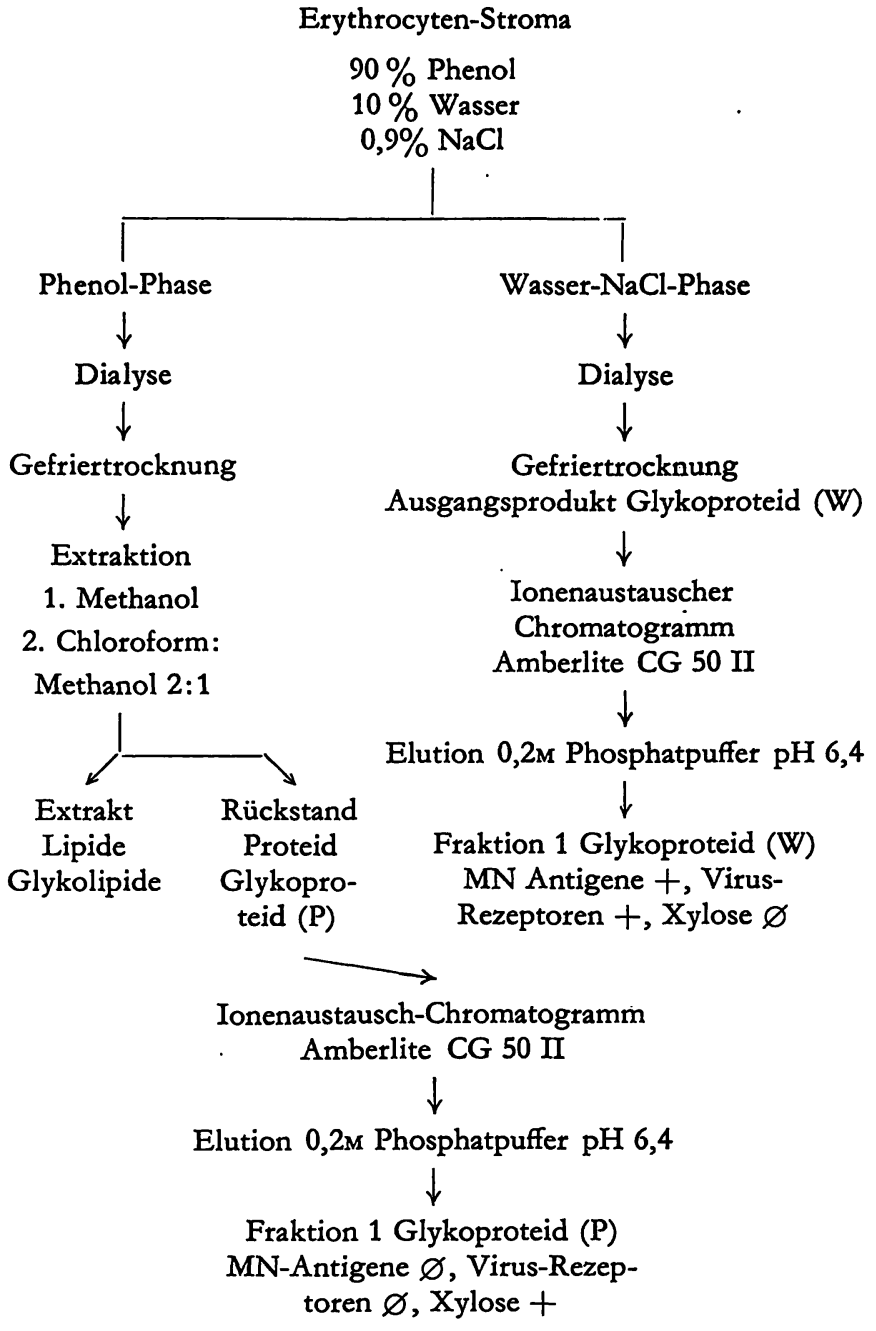

Abb. 1

Schema der Darstellung von Stroma-Glykoproteid (W) und (P)

machte folgende Vorbereitung èrforderlich: 24 stdg. Waschen des Amberlite CG 50 II in dest. Wasser, Entfernung des dest. Wassers durch Abnutschen, 4stdg. Waschen in Aceton, Entfernen des Acetons durch Abnutschen und Nachwaschen mit dest. Wasser. Die Überführung in die $\mathrm{H}^{+}$-Form erfolgte durch 4 stdg. Waschen des Austauschers mit 4N HCl. Nach Entfernung der $\mathrm{HCl}$ mit dest. Wasser wurde der Austauscher in 0,2M Natrium-Phosphatpuffer pH 6,4 gebracht. Nach 8 Stdn. Füllen der Säule $(\varnothing 0,8 \mathrm{~cm}$, Länge $30 \mathrm{~cm}$ ) und Spülen der Säule mit Phosphatpuffer bis ein konstanter pH-Wert von 6,4 erreicht war. Bei der feinen Korngröße muß die Aufschwemmung des Ionenaustauschers in den einzelnen Präparationsstufen unter ständigem Rühren vorgenommen werden. Nach Auftragen von $30-50 \mathrm{mg}$ Glykoproteid-Ausgangssubstanz in Phosphatpuffer-Lösung (1 $\mathrm{g} / 100 \mathrm{ml}$ ) erfolgt die stufenweise Elution mit

1. $0,2 \mathrm{M}$ Phosphatpuffër $\mathrm{pH} 6,4$

2. Puffergradient mit fallender Ionenstärke

3. $0,1 \mathrm{~N} \mathrm{HCl}$ und

4. 1 proz. Ammoniak.

Die Elutionsgeschwindigkeit betrug $1-2 \mathrm{ml}$ pro $5 \mathrm{Min}$. Die Registrierung der Fraktionen erfolgte mit dem LKB-Uvicord bei $254 \mathrm{~nm}$ und kolorimetrischer Bestimmung der Neuraminsäure in den einzelnen Fraktionen nach der Methode von SvenNerholm (29).

Folgende Einzeluntersuchungen wurden durchgeführt:

N-Bestimmung nach KJELDAHL,

Ermittlung der Gesamthexosen mit der Orcin- und AnthronMethode, bezogen auf einen Galaktose-Standard $(26,27)$,

Fucose-Bestimmung nach Dische und Shetrles (28),
Neuraminsäure-Bestimmung mit der Resorcin-Methode von SVENNERHOLM (29),

Bestimmung der enzymatisch freigesetzten Neuraminsäure nach der Methode von WARren (30),

Neuraminidase-Abbau mit $1 \mathrm{mg}$ (500IE) kristalliner Neuraminidase (aus Vibrio cholerae) (1 Ampulle), Behring-Werke, Marburg. Eingesetzt wurden $2 \mathrm{mg}$ Glykoproteid,

Bestimmung der Neuraminsäure an den Nativ-Erythrocyten nach der Methode von Trscher und Peters (31),

Glucuronsäure-Bestimmung nach Dische und ShETrLes, modifiziert von GREGORY $(32,33)$,

Xylose-Bestimmung mit der kolorimetrischen Methode von ROE und RICE (34), nach Hydrolyse des Glykoproteids mit 2N Schwefelsäure, anschließender Neutralisation der Schwefelsäure mit Bariumhydroxyd bei $50^{\circ}$. und Entfernung des restlichen Bariums an Dowex 50 X 8 (200-400 mesh), $\mathrm{H}^{+}$-Form,

Hexosamin-Bestimmung nach Hydrolyse des Glykoproteids mit 8 $\mathrm{N} \mathrm{HCl} \mathrm{(3} \mathrm{Stdn.} 100^{\circ}$ ) mit der Methode von Cesst (35). Der Neutralzucker-Verlust durch Hydrolyse wurde durch die Quote des Hydrolyse-Verlustes der Reinsubstanz korrigiert.

\section{Chromatographische Untersuchungen}

Zur Chromatographie der Neutralzucker wurden $2 \mathrm{mg}$ Glykoproteid mit $1 \mathrm{ml} 2 \mathrm{~N}$ Schwefelsäure bei $100^{\circ} 4 \mathrm{Stdn}$. hydrolysiert, und die Schwefelsäure mit Bariumhydroxyd neutralisiert. Das Hydrolysat wurde auf ein kleines Volumen eingeengt und in Streifen auf Cellulose-Dünnschichtplatten aufgetragen. Als bestes,Lösungsmittel-System bewährte sich das von VomнoF und TUCKER (36) beschriebene Lösungsmittel tert. Butanol/Methyläthylketon/Ameisensäure/Wasser $=8: 6: 3: 3(\nabla / v)$ bei zweimaligem Entwickeln der Platte. Die Zucker wurden mit Anilinphthalat sichtbar gemacht. Die quantitative Zuckerbestimmung wurde nach chromatographischer Trennung auf Papier mit 2proz. Triphenyltetrazoliumchloridlösung in $0,5 \mathrm{~N} \mathrm{NaOH}$ (37) vorgenommen, die eine Fehlerbreite von $5-8 \%$ hat. Die Nachweisgrenze lag bei $15-20$ $\mu \mathrm{g}$ der einzelnen Zuckerkomponenten. Die Untersuchungen mit verschiedenen Hydrolyse-Methoden (80proz. Ameisensäure, 50proz. Trichloressigsäure, Ionenaustauscher Dowex und Amberlite in H-Form) zeigten, daß diese der Technik mit 2N Schwefelsäure nicht überlegen waren. Vergleichende chromatographische Untersuchungen auf verschiedenen Cellulose-Sorbentien und Papier zeigten keine besseren Trennergebnisse als diejenigen, die wir auf Cellulose-Fertigplatten (Merck) erhalten hatten.

Die chromatographische Neuraminsäure-Identifizierung wurde nach enzymatischer Hydrolyse mit Neuraminidase (1 mg (500 IE), Neuraminidase Behring-Werke Marburg), Dialyse der Neuraminsäure und Entsalzung des Dialysates über Ionenaustauscher Dowex-H ${ }^{+} 50 \times 8$ (200-400 mesh) auf Cellulose-Dünnschichtplatten im Laufmittel nButanol/nPropanol/0,1 $\mathrm{HCl}=1: 2: 1$ (v/v) durchgeführt (15). Der Nachweis erfolgte mit alkalischem Silbernitrat und Brals-Reagenz.

Die Aminozucker wurden nach Hydrolyse des Glykoproteids mit $8 \mathrm{~N} \mathrm{HCl}\left(3 \mathrm{Stdn}\right.$. bei $100^{\circ}$ ) nach Entfernūng der $\mathrm{HCl}$ durch Stickstoff zur Trockene gebracht und chromatographisch auf Papier in dem Lösungsmitttelsystem von FISCHER und NebEL (38) nach 18 stdg. Chromatographie mit alkalischem Silbernitrat nachgewiesen. Diese Identifizierung der Aminozucker wurde durch den Ninhydrin-Abbau des Galaktosamins zu Lyxose und des Glucosamins zu Arabinose chromatographisch auf Cellulose-Dünnschichtplatten in dem Laufmittel von VoMHOF und TUCKER (36) bestätigt. Die Chromatogramme der Aminosäuren wurden auf CelloluseDünnschichtplatten im Kleinformat $7,4 \times 7,4 \mathrm{~cm}$ in Schieferdecker-Färbeküvetten, die in Kammern von $10 \times 10 \times 15 \mathrm{~cm}$ gebracht wurden, angefertigt. Hierzu wurde $1 \mathrm{mg}$ Glykoproteid in $1 \mathrm{ml} 6 \mathrm{~N} \mathrm{HCl} 24$ Stdn. hydrolysiert, die Salzsäure mit Stickstoff vertrieben, das trockene Hydrolysat in $0,01 \mathrm{~m} l$ Wasser aufgenommen und $0,001 \mathrm{ml}$ aufgetragen. Das Lösungsmittel-System bestand aus nButanol/Eisessig/Wasser $=12: 3: 5(\nabla / v)$ für die 1 . Dimension und Phenol/Ammoniak/Wasser $=640: 3,4: 144(\nabla / v)$ für die 2. Dimension. Die Aminosäuren wurden mit 0,2proz. NinhydrinLösung in Aceton bei $100^{\circ}$ dargestellt. 
Dic Überprüfung des Reinbeitsgrades der Glykoproteide erfolgt mit Elektrophorese auf Cellulose-Acetat-Folien in 0,2M Veronal-Puffer pH 8,2 und der Disk-Elektrophorese auf Polyacrylamid-Gel in einem Tris-Puffer-System $\mathrm{pH} 8,9$ und 6,9. Die Darstellung erfolgte mit Amidoschwarz.

\section{Ergebnisse}

Das MN-Antigen-haltige Stroma-Glykoproteid (W) wurde nach der Methode von Westphal und Mitarbeitern (25), modifiziert von UHLENBRUCK und SCHMID (15) aus der $\mathrm{NaCl}$-haltigen wäßr. Phase isoliert. Das Glykoproteid $(\mathrm{P})$, das sich in seinen chemischen und serologischen Eigenschaften von dem Glykoproteid (W) eindeutig unterscheidet, konnte aus der Phenolphase der Stroma-Präparation dargestellt werden, nachdem die Lipide mit organischen Lösungsmitteln extrahiert worden waren. Das Glykoproteid (P) wurde aus dem trokkenen lipidfreien Extraktrückstand mit Wasser ausgezogen. Die Glykoproteide (W) und (P) wurden durch Ionenaustauscher-Chromatographie an einer AmberliteSäule CG 50 II durch stufenweise Elution mit Phosphatpuffer $\mathrm{pH}$ 6,4 und Phosphatpuffer-Gradienten in fallender Ionenstärke gereinigt. Diese Technik war der Reinigung durch Sephadex-Gel-Filtration überlegen, bei der wir mehrere Fraktionen erhielten, die sich in ihrer Zusammensetzung jedoch nur geringfügig unterschieden (Abb. 2, 3). Bei der Ionenaustauscher-Säulenchromatographie wurde das neuraminsäurereiche Glykoproteid (W) und das Xylose-haltige Glykoproteid (P) in getrennten Läufen jeweils in der Hauptfraktion, die mit 0,2M Phosphatpuffer eluiert worden war, dargestellt. Die Ausbeute an gereinigtem Glykoproteid betrug $60-65 \%$ des Ausgangsmaterials. Sie war jedoch ebenso wie die an Erythrocyten-Stroma von den AB0-Eigenschaften der Erythrocyten und der Lagerungszeit der Blutkonserven abhängig. Bei 0-Erythrocyten war die Ausbeute an Glykoproteid am größten, wir fanden
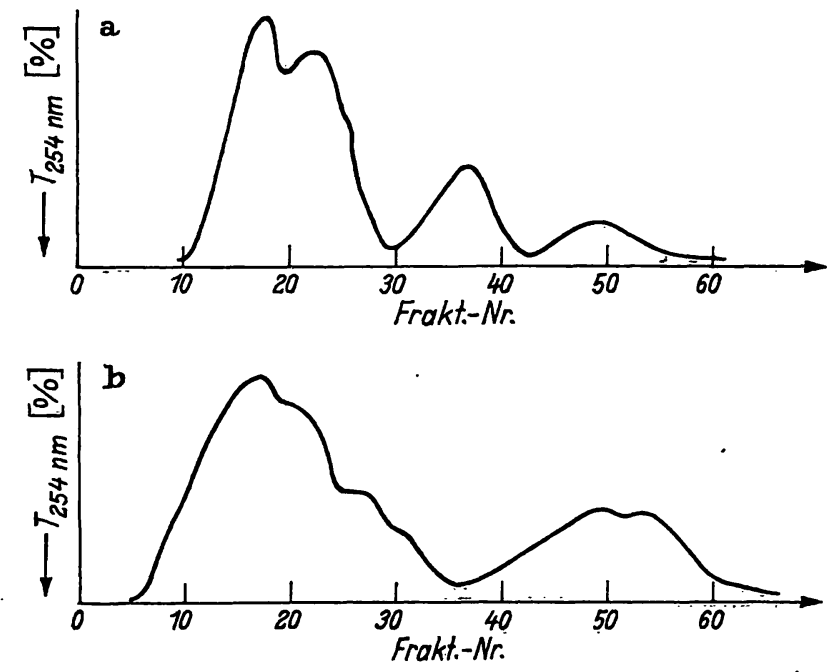

Abb. 2

Fraktionierung des MN-haltigen Stroma-Glykoproteids (W) an Sephadex Gel G 75. Elutionsmittel: Wasser; Elutionsgeschwindigkeit: $1 \mathrm{~m} l / \mathrm{Min}$. Säulenlänge $40 \mathrm{~cm}, \varnothing 2,5 \mathrm{~cm}$.

a) $140 \mathrm{mg}$ Glykoproteid aus gepoolten Konserven verschiedener Blutgruppen

b) $150 \mathrm{mg}$ Glykoproteid aus einer Konserve Blutgruppe 0
2,5-4,0\%, berechnet auf die Stromamenge. Die Stromaausbeute, bezogen auf das Erythrocyten-Trockengewicht, betrug 2-3\%. Längere Lagerung der Blutkonserven bedingte eine erhebliche Reduktion dieser Ausbeute, die für Stroma und Glykoproteid bei Lagerungszeiten über 20 Tage $40-50 \%$ ausmachen konnte (Tab. 1). Die quantitative Ausbeute bei AB-Erythrocyten unterschied sich nicht wesentlich von derjenigen bei 0-Erythrocyten. Bei A- und B-Blutgruppeneigenschaften hingegen war auch bei kurzfristiger Lagerungszeit die Glykoproteid-Menge erheblich herabgesetzt und wurde besonders bei den B-Erythrocyten durch Lagerungszeiten über 20 Tage noch stärker reduziert (Tab. 1, 2). Die MN-Eigenschaften hatten keinen Einfluß auf die Glykoproteid-Ausbeute, waren jedoch für die quantitative Kohlenhydrat-Zusammensetzung und besonders für den Neuraminsäure-Gehalt des Glykoproteids (W) bedeutungsvoll. Rh-Eigenschaften beeinflußten die Ausbeute und die Kohlenhydratzusammensetzung des Glykoproteids (W) nicht. Im Gegensatz zu dem Glykoproteid (W) wurde die Menge des Glykoproteids (P) von den $A B 0$-Eigenschaften der Erythrocyten und der Lagerungszeit kaum beeinflußt. Hier fanden wir jedoch eine Abhängigkeit der prozentualen KohlenhydratZusammensetzung von dem $\mathrm{AB} 0-$ System. Hingegen hatten die MN-Eigenschaften keinen Einfluß auf den

Tab. 1

Ausbeute des MN-Antigen-haltigen Glykoproteids (W) bei verschieden langer Lagerungszeit der Konserven

\begin{tabular}{|c|c|c|c|}
\hline Lagerungszeit in Tagen & $1 \frac{5}{5}^{5}$ & $5-15$ & $\begin{array}{l}20 \\
10\end{array}$ \\
\hline \multirow{6}{*}{$\begin{array}{l}\text { Erythrocyten eingesetzt (g) } \\
\text { Stroma-Glykoproteid (W) in mg } \\
\text { Glykoproteid (W) in \% des } \\
\text { Erythrocyten-Gewichts } \\
\text { Neuraminsäure des Glykoproteids } \\
\text { (W) in mg } \\
\text { Neuraminsäure des Nativ- } \\
\text { Erythrocyten in mg } \\
\text { Neuraminsäure in \% des Glyko- } \\
\text { proteids (W) } \\
\text { Neuraminsäure in \% der Nativ- } \\
\text { Erythrocyten. }\end{array}$} & $\begin{array}{l}63 \\
54\end{array}$ & $\begin{array}{l}65 \\
36\end{array}$ & $\begin{array}{l}50 \\
16\end{array}$ \\
\hline & 0,08 & 0,06 & 0,03 \\
\hline & 10,6 & 7,3 & 3,3 \\
\hline & 45,2 & 56,3 & 35,4 \\
\hline & 0,016 & 0,011 & 0,006 \\
\hline & 0,07 & 0,085 & 0,07 \\
\hline
\end{tabular}

Kohlenhydratgehalt und die Verteilung der Kohlenhydrat-Komponenten (Tab. 3, 4).

Die immunologischen Untersuchungen mit Immunodoppel-Diffusion und Immun-Elektrophorese sowie die Reinheitsprüfung der Glykoproteide auf CelluloseAcetat-Folien und mit der Disk-Elektrophorese zeigten nach Reinigung der Ausgangssubstanz keine Beimengung von Serum-Proteinen (Abb. 4). In der DiskElektrophorese allerdings konnten wir sowohl bei Glykoproteid $(\mathrm{W})$ und $(P)$ noch 2 Banden mit Amidoschwarz darstellen, ein Befund, der jedoch bei dieser Technik nicht als sicheres Zeichen von chemischer Heterogenität zu deuten ist, da wir auch bei immunologisch reinen Serum-Proteinen oft mit der Disk-Technik noch mehrere Fraktionen beobachten konnten. Dies erklärt sich wahrscheinlich durch Proteinaggregatbildung immunologisch identischer Proteine, die aufgrund der unterschiedlichen Aggregatgrößen durch den Siebeffekt des Acrylamid-Gels getrennt werden. 

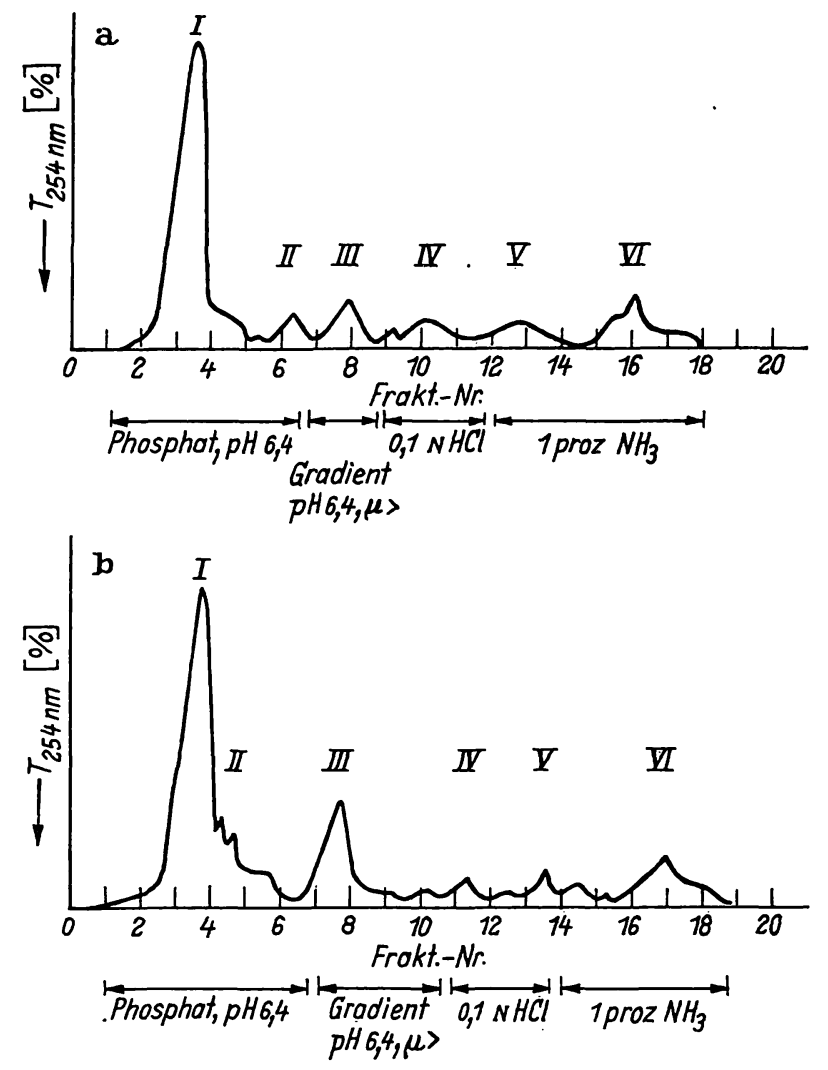

d) Glykoproteid (P)

Passage an Ionenaustauscher Amberlite CG 50 II. Säule $30 \mathrm{~cm}$ lang, $\varnothing 0,8 \mathrm{~cm}$. Elutionsgeschwindigkeit $2 \mathrm{ml} / 5 \mathrm{Min}$.

Elutionsmittel:

1. $0,2 \mathrm{M}$ Phosphatpuffer, $\mathrm{pH} 6,4$

2. Phosphatpuffergradient $\mathrm{pH} 6,4$ fallender Ionenstärke, Anfangskonzentration $0,2 \mathrm{M}$

3. $0,1 \mathrm{~N} \mathrm{HCl}$

4. 1 proz. Ammoniak

Es wurden folgende Konserven untersucht:

a) Konserve 14 (W) Blutgruppe $0 \mathrm{Rh}(+) \mathrm{N}, \mathrm{S}(+)$

$\begin{array}{lcccccc}\text { Fraktion } & \text { I } & \text { II } & \text { III } & \text { IV } & \text { V } & \text { V1 } \\ \text { Ausbeute in mg } & 24 & 1 & 2 & 0,6 & 0,4 & 2 \\ \text { MN Antigen } & + & (+) & (+) & \varnothing & \varnothing & \varnothing \\ \text { Virusrezeptor } & + & (+) & (+) & \varnothing & \varnothing & \varnothing \\ \text { N-Acetyl- } & & & & & & \\ \text { Neuraminsäure } & ++ & + & (+) & \varnothing & \varnothing & \varnothing\end{array}$

b) Konserve 9 (W) Blutgruppe $0 \mathrm{Rh}(+) \mathrm{MN}, \mathrm{S}(-)$

$\begin{array}{lcccccc}\text { Ausbeute in mg } & 15 & 4 & 6 & 2 & 0,6 & 2 \\ \text { MN Antigen } & + & (+) & (+) & \varnothing & \varnothing & \varnothing \\ \text { Virusrezeptor } & + & (+) & (+) & \varnothing & \varnothing & \varnothing \\ \text { N-Acetyl- } & & & & & & \\ \text { Neuraminsäure } & ++ & + & (+) & \varnothing & \varnothing & \varnothing\end{array}$

c) Konserve 13 (W) Blutgruppe $0 \mathrm{Rh}(+) \mathrm{M}, \mathrm{S}(-)$

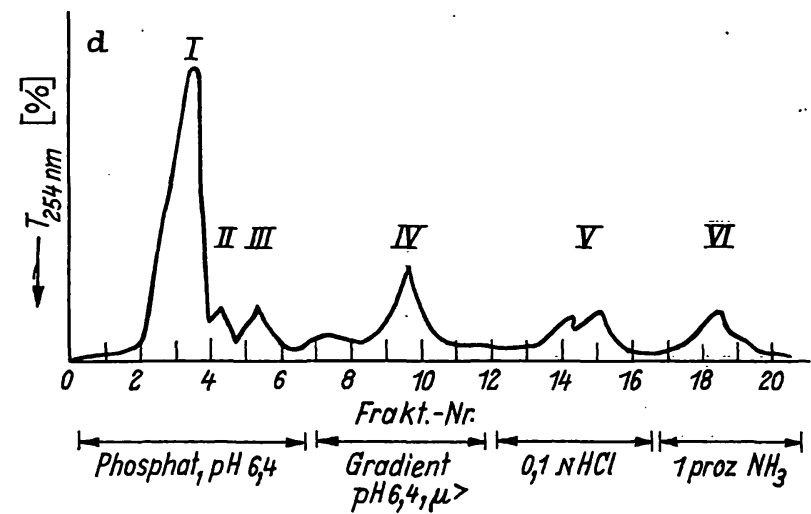

\begin{tabular}{|c|c|c|c|c|c|c|}
\hline Ausbeute in $\mathrm{mg}$ & 22,5 & 2,5 & 2,4 & 0,1 & 0,4 & 2,5 \\
\hline MN Antigen & + & $(+)$ & $(+)$ & $\varnothing$ & $\varnothing$ & $\varnothing$ \\
\hline $\begin{array}{l}\text { Virustezeptor } \\
\text { N-Acetyl- }\end{array}$ & + & $(+)$ & $(+)$ & $\varnothing$ & $\varnothing$ & $\varnothing$ \\
\hline Neuraminsäure & ++ & + & $\varnothing$ & $\varnothing$ & $\varnothing$ & $\varnothing$ \\
\hline \multicolumn{7}{|c|}{ d) Konserve $13(\mathrm{P})$ Blutgruppe $0 \mathrm{Rh}(+) \mathrm{M}, \mathrm{S}(-)$} \\
\hline Ausbeute & 19 & 0,7 & 7,0 & 7,0 & 0,5 & $C_{5}$ \\
\hline MN Antigen & $\varnothing$ & $\varnothing$ & $\varnothing$ & $\varnothing$ & $\varnothing$ & \\
\hline $\begin{array}{l}\text { Virusrezeptor } \\
\text { N-Acetyl- }\end{array}$ & $(+)$ & $\varnothing$ & $\varnothing$ & $\varnothing$ & $\varnothing$ & \\
\hline Neuraminsäure & + & $(+)$ & $(+)$ & $\varnothing$ & $\varnothing$ & \\
\hline
\end{tabular}

Tab. 2

A bhängigkeit der Glykoproteid-Ausbeute und des Neuraminsäuregehaltes von Glykoproteid und Nativ-Erythrocyten von AB0-Blutgruppeneigenschaften und unterschiedlich langer Lagerungsz̃eit der Blutkonserven

\begin{tabular}{|c|c|c|c|c|}
\hline \multirow[t]{2}{*}{ Blutgruppe } & \multirow[t]{2}{*}{ Lagerungsdauer } & \multirow{2}{*}{$\begin{array}{l}\text { Stroma-Glykoproteid } \\
\text { in \% des } \\
\text { Erythrocytengewichts }\end{array}$} & \multicolumn{2}{|c|}{ Neuraminsäure } \\
\hline & & & $\begin{array}{l}\text { in } \% \text { des } \\
\text { Stroma-Gilykoproteids }\end{array}$ & $\begin{array}{l}\text { in \% der } \\
\text { Nativ-Erythrocyten }\end{array}$ \\
\hline $\begin{array}{l}A B \\
A \\
B \\
0 \\
A B \\
A \\
B \\
0\end{array}$ & $\begin{array}{c}1-5 \text { Tage } \\
\text { über } 20 \text { Tage }\end{array}$ & $\begin{array}{l}0,09 \\
0,05 \\
0,05 \\
0,074 \\
0,04 \\
0,032 \\
0,015 \\
0,05\end{array}$ & $\begin{array}{l}0,018 \\
0,01 \\
0,01 \\
0,015 \\
0,008 \\
0,007 \\
0,063 \\
0,010\end{array}$ & $\begin{array}{l}0,08 \\
0,06 \\
0,06 \\
0,07 \\
0,1 \\
0,06 \\
0,03 \\
0,08\end{array}$ \\
\hline
\end{tabular}


Tab. 3

Prozentualer Anteil der Kohlenhydrat-Komponenten und des Proteinanteils von Fraktion 1 des Glykoproteids (W) mit verschiedenen MN-AntigenEigenschaften nach Reinigung durch Ionenaustausch-Chromatographie an Amberlite CG 50 II. Glykoproteid (W) isoliert aus Erythrocyten der Blutgruppe $0, M, R h(+) ; 0, M N, R h(+) ; 0, N, R h(+) ; A B, N, R h(+)$ und $A B, N, r h(-)$.

\begin{tabular}{|c|c|c|c|c|c|}
\hline & $0, M \mathrm{Rh}(+)$ & $0, M N$ Rh $(+)$ & $0, \mathrm{~N} R \mathrm{Rh}(+)$ & $A B, N$ Rh $(+)$ & $\mathrm{AB}, \mathrm{N}$ rh $(-)$ \\
\hline $\begin{array}{l}\text { Neuraminsäure } \\
\text { Hexosen } \\
\text { Hexosamin } \\
\text { Fucose } \\
\text { Glucuronsäure } \\
\text { Gesamtkohlenhydrate } \\
\text { Proteinanteil }\end{array}$ & $\begin{array}{r}23,0 \\
10,3 \\
8,2 \\
1,5 \\
0,5 \\
43,5 \\
47,0\end{array}$ & $\begin{array}{r}19,0 \\
14,5 \\
6,8 \\
0,5 \\
1,0 \\
41,8 \\
44,0\end{array}$ & $\begin{array}{r}18,0 \\
14,0 \\
9,0 \\
0,6 \\
1,5 \\
43,1 \\
46,0\end{array}$ & $\begin{array}{r}17,0 \\
16,0 \\
8,3 \\
0,6 \\
1,6 \\
43,5 \\
51,0\end{array}$ & $\begin{array}{r}17,0 \\
14,0 \\
8,0 \\
0,6 \\
1,0 \\
40,6 \\
50,0\end{array}$ \\
\hline
\end{tabular}
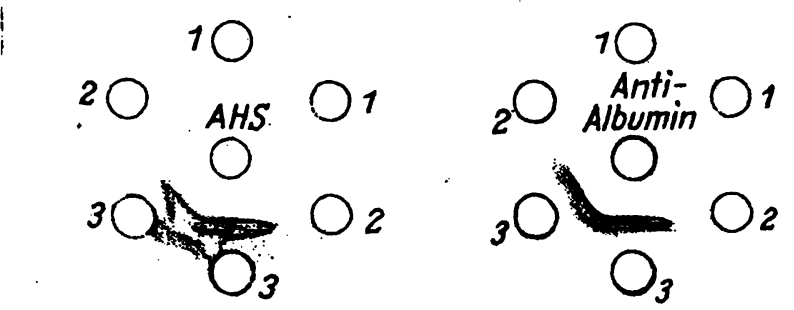

Abb. 4

Immundiffusion nach OuchterLoNY des Glykoproteids (W) vor (3) und nach $(1,2)$ Ionenaustauscher-Säulenpassage, links polyvalentes Human-Antiserum, rechts Antialbumin

Tab. 4

Vergleich der prozentualen Anteile der Kohlenhydrat-Komponenten des MN-Antigen-haltigen Glykoproteids (W) und des Xylose-haltigen des $M N$-Antigen-haltigen Glykoproteids (W) und des Xylose-haltigen durch Ionenaustausch-Chromatographie an Amberlite $C G 50$ II
durat

\begin{tabular}{lcc}
\hline & Glykoproteid. $(W)$ & Glykoproteid (P) \\
\hline Neuraminsäure & 23.0 & 11,0 \\
Hexosen & 10,3 & 13,0 \\
Hexosamin & 8,2 & 6,0 \\
Fucose & 1,5 & 0,5 \\
Glucuronsäure & 0,5 & 1,0 \\
Xylose & nicht & 3,5 \\
Gesamtkohlenhydrate & nachweisbar & 35,0 \\
Proteinanteil & 43,5 & 59,0 \\
\hline
\end{tabular}

Die mit kolorimetrischen und chromatographischen Methoden ermittelten Kohlenhydratkomponenten hatten bei dem gereinigten Glykoproteid (W) einen Gesamtgehalt von $37-41 \%$, der um etwa $3-4 \%$ höher lag als bei der Ausgangssubstanz. Chromatographisch war auf Cellulose-Dünnschichtplatten Galaktose, Mannose und Fucose in einer Verteilung von $4: 2: 1 \mathrm{zu}$ identifizieren (Abb. 5). Die Neuraminsäure, die für MN-Antigene und Virus-Rezeptoren des Glykoproteids (W) die determinierende Kohlenhydratkomponente ist, hatte in dem gereinigten Glykoproteid (W) eine Konzentration von $17-23 \%$ gegenüber $14-18 \%$ in der Ausgangssubstanz. Sie war mit Neuraminidase zu 85-90\% abspaltbar und konnte chromatographisch als N-Acetyl-Neuraminsäure. identifiziert werden. Bei Erythrocyten mit M-Eigenschaften fanden wir die höchsten Neuraminsäure-Konzentrationen, wie aus Vergleichsuntersuchungen von 0-Erythrocyten mit verschiedenen $\mathrm{M}-\mathrm{N}$ - und $\mathrm{MN}$-Antigenen zu ersehen war (Tab. 3). Hingegen war bei Erythrocyten mit M-Antigenen die Hexosekonzentration etwas niedriger, die Fucose zeigte bei verschiedenen MN-Fraktionen keine Differenz. Die Hexosamin-Bestimmung wurde nach der Methode von CESSI (35) durchgeführt, da mit dieser Technik die Aminozuckerchromogen-Bildung durch farbgleiche Chromogene aus Hexosen in Gegenwart von Aminosäure oder Ammoniak vermieden wird. Der Hexosamingehalt lag um $6-8 \%$ und war bei Erythrocyten mit MN-Eigenschaften etwas niedriger als bei M- und N-Erythrocyten. Durch die Faktoren des $A B 0-S y s t e m s$ wurde er in seiner Konzentration nicht beeinflußt (Tab. 3). Nach der chromatographischen Trennung der aus den Glykoproteiden (W) durch Hydrolyse freigesetzten Hexosamine konnten wir papierchromatographisch eine Verteilung von Galaktosamin: Glucosamin $=5: 3$ feststellen. Durch den NinhydrinAbbau (39) der Aminozucker zu den entsprechenden Pentosen Lyxose bzw. Arabinose wurde dieser Befund bestätigt. Die Glucuronsäure-Konzentration war bei allen Erythrocyten etwa gleich hoch, und lag zwischen $1-1,5 \%$.

In dem Virus-Hämagglutinations-Test und bei der serologischen Bestimmung der MN-Antigene konnten die Virus-Rezeptor- und die MN-Antigen-Eigenschaften bei der Glykoproteid-Ausgangssubstanz und dem gereinigten Glykoproteid (W) nachgewiesen werden. Nach enzymatischer Hydrolyse der Neuraminsäure mit kristal-

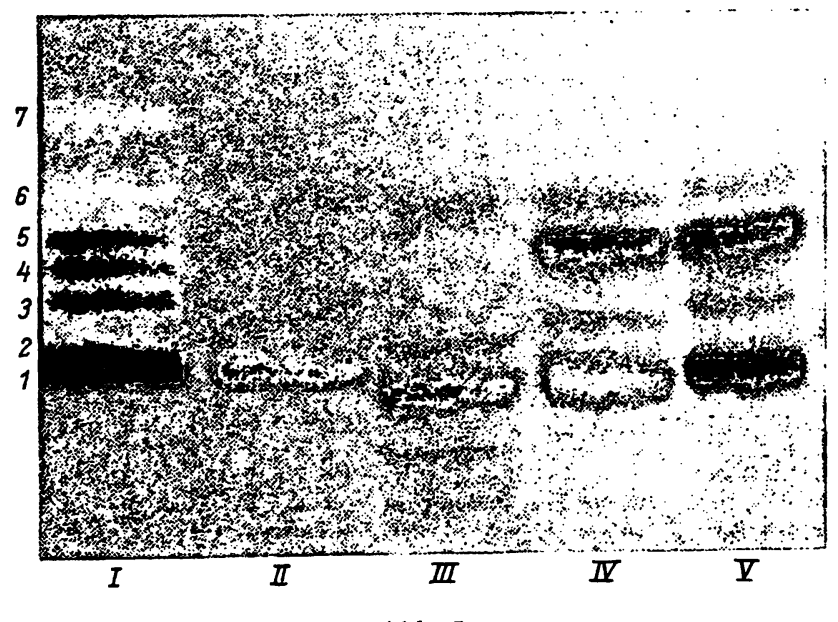

Abb. 5

Cellulose-Dünnschicht-Chromatographie des MN-Antigen-haltigen Glykoproteid (W)- und des Xylose-haltigen Glykoproteid (P)Hydrolysates.

Laufmittel: tert. Butanol/Methyläthylketon/Ameisensäure/Wasser $=8: 6: 3: 3(\mathrm{v} / \mathrm{v})$, zweimaliges Entwickeln derselben Platte, Darstellung mit Anilinphthalat bei $100^{\circ} 10 \mathrm{Min}$.

I. Standard: 1. Galaktose, 2. Glucose, 3. Mannose, 4. Arabinose, 5. Xylose, 6. Fucose, 7. Rhamnose. - II. Glykoproteid (W) Ausgangssubstanz. - III. Glykoproteid (W) nach Reinigung über Ionenaustauscher Amberlite CG 50 II. - IV. Glykoproteid (P) Ausgangssubstanz. - V. Glykoproteid (P) nach Reinigung über Ionenaustauscher Amberlite CG 50 II. 
liner Neuraminidase war sowohl die Virus-Hämagglutinations-Hemmung als auch die M-Antigen-Eigenschaft aufgehoben. Mit der zweidimensionalen Chromatographie auf Cellulose-Dünnschicht-Platten, die bei der Mikro-Methode zahlreiche Paralleluntersuchungen in verschiedenen Lösungsmittel-Systemen und mit verschiedenen Nachweisverfahren erlaubte, konnten in dem Glykoproteid (IV) 16 Aminosäuren identifiziert werden (Abb. 6). In der Ausgangssubstanz fanden wir Asparaginsäure, Glutaminsäure, Serin, Glycin, Alanin, Threonin, Tyrosin, Valin, Isoleucin, Leucin, Prolin, Histidin, Lysin, Phenylalanin, Arginin und Cystein. In dem gereinigten Glykoproteid (W) war Cystein nicht mehr nachweisbar. Serin zeigte den intensivsten NinhydrinFleck und konnte auch bei Verwendung von NinhydrinZinnchlorid-Spray, der vergleichbare Ninhydrin-Intensitäten bei verschiedenen Aminosäuren ergibt, als die Aminosäure bestätigt werden, die in höchster Konzentration vorliegt. driger als in dem Glykoproteid (W), bei AB- und A, BErythrocyten lag der Gesamthexosegehalt etwas höher, so daß die Differenzen in dem Gesamtkohlenhydratgehalt bei diesen Blutgruppen gegenüber dem Glykoproteid (W) weniger zum Ausdruck kamen. Neben Xylose war Galaktose, Mannose, Fucose deutlich und Glucose in Spuren nachweisbar. Die Zucker hatten eine Verteilung von Galaktose:Mannose:Fucose:Xylose: Glucose = 4:2:1:3:1 (Abb. 5). Das Galaktosamin: Glucosamin-Verhältnis des Glykoproteids (P) betrug bei 0-Erythrocyten 3:2. Diese Aminozucker konnten durch den Ninhydrin-Abbau zu Lyxose bzw. Arabinose bestätigt werden. Die Neuraminsäure, deren Konzentration unabhängig von dem $\mathrm{AB} 0$ - und $\mathrm{MN}$-System bei dem Glykoproteid $(P)$ niedriger lag als bei dem Glykoproteid (W), war ebenfalls als N-Acetyl-Neuraminsäure zu identifizieren. Sie konnte jedoch nur partiell zu $60-70 \%$ abgespalten werden. Die Aminosäure-Zusammensetzung des Glykoproteids (P) zeigte in der qualita-
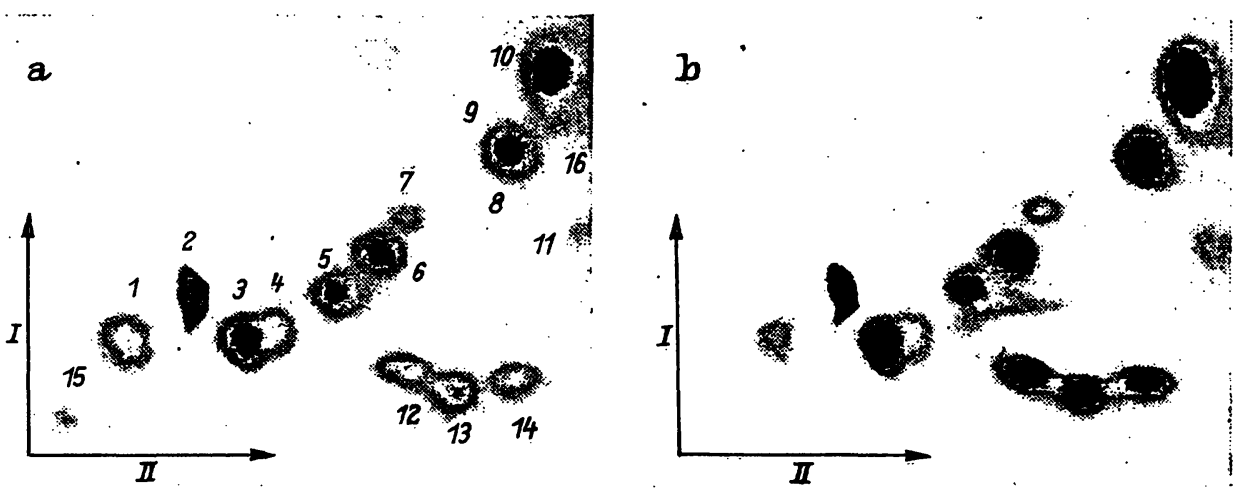

Abb. 6

Zweidimensionales Aminosäure-Chromatogramm des Glykoproteid (W)-Hydrolysates auf Cellulose-Dünnschichtplatten. a) Ausgangssubstanz, b) nach Reinigung durch Ionenaustauscherpassage, Laufmittel: 1. Dimension: $\mathrm{nButanol} /$ Eisessig/Wasser $=$ 12:3:5 ( $\mathrm{v} / \mathrm{v})$, 2. Dimension: Phenol/Ammoniak/Wasser $=640: 3,4: 144$ (v/v). Darstellung mit 0,2proz. Ninhydrin in Aceton. 1. Asparaginsäure, 2. Glutaminsäure, 3. Serin, 4. Glycin, 5. Threonin, 6. Alanin, 7. Tyrosin, 8. Valin, 9. Leucin, 10. Phenylalanin, 11. Prolin, 12. Histidin, 13. Lysin, 14. Arginin, 15. Cystein, 16. Isoleucin.

Das Xylose-haltige Glykoproteid $(P)$ aus der Phenolphase besaß keine MN-Antigen-Eigenschaften und die VirusHämagglutination wurde durch das Glykoproteid $(P)$ weniger gehemmt als durch Glykoproteid (W). Die Ausbeute an Glykoproteid (P) war um 20-25\% geringer als bei Glykoproteid (W). Die Löslichkeitsverhältnisse waren gleich und die Reinigung durch IonenaustauscherChromatographie entsprach der von Glykoproteid (W). Bei 0,M-Erythrocyten war der Gesamtkohlenhydratgehalt mit 26-30\% etwas niedriger und der Peptidgehalt höher als bei dem Glykoproteid (W) (Tab. 3). Der wesentlichste chemische Unterschied war jedoch durch den Nachweis von Xylose gegeben, die in einer Konzentration von $3-3,5 \%$ vorlag. Diese Pentose konnte durch Cellulose-, Dünnschicht-, Papier- und gaschromatographische Untersuchungen des Tri-Methylsilyl-Derivates identifiziert werden und mit Bromanilin nach der Methode von ROE und RICE (34) kolorimetrisch bestimmt werden. Die Neuraminsäure- und Hexosamin-Konzentration lag bei 0 -Erythrocyten nie-

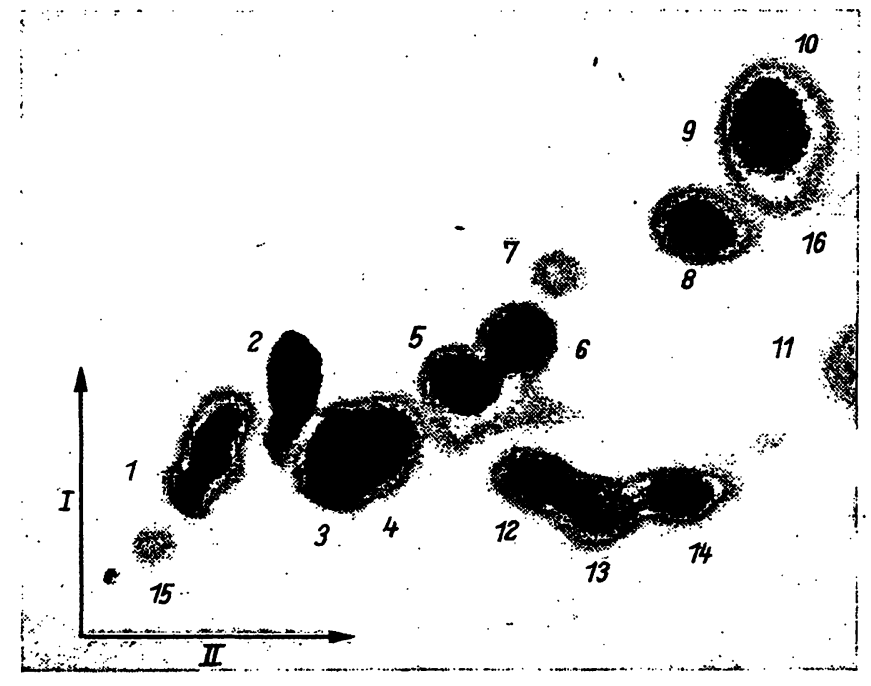

Abb. 7

Zweidimensionales Aminosäure-Chromatogramm des Glykoproteid (P)-Hydrolysates auf Cellulose-Dünnschichtplatten. Technik, Lösungsmittel und Aminosäure-Verteilung s. Abb. 6* 
tiven chromatographischen Untersuchung keine sicheren Unterschiede gegenüber der Aminosäure-Zusammensetzung von Glykoproteid (W) (Abb. 7).

Immunologisch war nach Reinigung der Ausgangssubstanz keine Präzipitation mit Antihuman-Serum feststellbar. Bei einheitlichen Banden an der CelluloseAcetat-Folie waren auch bei dem Glykoproteid $(P)$ in der Disk-Elektrophorese zwei Banden mit Amidoschwarz darzustellen. Aufgrund der geringeren Konzentration endständiger Neuraminsäure und der geringeren enzymatisch abspaltbaren Neuraminsäure-Quote zeigte das Glykoproteid ( $P$ ) eine geringere Hemmung der Virus-Hämagglutination als Glykoproteid (W).

\section{Diskussion}

Die Isolierung des MN-haltigen Glykoproteids (W) der Erythrocyten-Membran hat gezeigt, daß die Ausbeute dieses Glykoproteids sowohl von AB0-Eigenschaften als auch von der Lagerungszeit der Blutkonserven abhängig ist. Die Glykoproteid-Ausbeute war bei 0 - und AB-Erythrocyten am höchsten und zeigte auch bei diesen Blutgruppen die geringste Reduktion nach langer Lagerungszeit. Diese Beobachtung ist für die Beurteilung der Funktionstüchtigkeit infundierter Erythrocyten von Bedeutung, da sie erkennen läßt, daß schon vor Ablauf der durch Chrommarkierung ermittelten Überlebenszeit der Erythrocyten blutgruppenabhängige strukturelle Veränderungen an der Membran ablaufen,

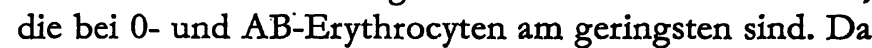
wir auch in Paralleluntersuchungen zu dem Neuraminsäuregehalt des Nativ-Erythrocyten fanden, daß nicht nur die isolierte Glykoproteidmenge, sondern auch der Neuraminsäuregehalt des Nativ-Erythrocyten von ABOEigenschaften und der Zeitdauer der Konservenlagerung abhängig ist, haben diese Befunde praktische Bedeutung. Bei Bluttransfusionen mit dem Ziel der ErythrocytenSubstitution sollte man deshalb bei Konserven mit der Blutgruppe A und B möglichst kurze Lagerungszeiten der Blutkonserven anstreben.

Entgegen anderen Autoren (23) fanden wir bei MErythrocyten einen höheren Neuraminsäuregehalt als bei MN- und N-Erythrocyten. Diese Werte erscheinen glaubhaft, da M-Erythrocyten auch N-Antigene enthalten, die durch partielle enzymatische NeuraminsäureAbspaltung wie in den Untersuchungen von SPRINGER, NAGAI und StaldER $(21,22)$ gezeigt wurde, freigelegt werden können. Diese Autoren fanden ebenfalls bei M-Erythrocyten einen höheren Neuraminsäure-Wert (21). Die Reinigung der Glykoproteid (W)=Ausgangssubstanz durch Ionenaustauscher-Chromatographie liefert ein Untersuchungsmaterial, bei dem reproduzierbare Analysen-Daten der Neuraminsäure, Hexosen, Fucose, Hexosamin, Aminosäuren und der prozentualen Verteilung der Hexosen und Pentosen zu erhalten waren. Trotz immunologischer Homogenität fanden wir in der Disk-Elektrophorese noch die Auftrennung in zwei Banden, so daß der theoretische Homogenitätșanspruch noch nicht vollkommen erfüllt zu sein scheint. Neben der Galaktose als Hauptkomponente der Neutralzucker fanden wir Mannose und Fucose, die auch von anderen Autoren beschrieben wurde. Das Vorkommen von zwei Hexosaminen, bei denen in dem Glykoproteid (W) das Galaktosamin überwiegt, konnte chromatographisch nachgewiesen und durch den Ninhydrin-Abbau zu den korrespondierenden Pentosen bestätigt werden. Die von allen Autoren übereinstimmend als N-Acetyl-Neuraminsäure identifizierte Neuraminsäure bei Menschen-Erythrocyten, die für die MN-Antigen- und Virus-RezeptorEigenschaften die entscheidende Kohlenhydratgruppe darstellt, konnte nach enzymatischer Freisetzung auch in unseren Untersuchungen chromatographisch bestätigt werden. Thre $85 \%$ ige Abspaltung bei dem Glykoproteid (W) konnte sowohl durch die Neuraminsäure-Bestimmung nach erfolgter Freisetzung der Neuraminsäure durch Neuraminidase mit der Warren-Methode als auch durch die Bestimmung der gebundenen Neuraminsäure nach SvenNerholm in dem enzymatisch vorbehandelten Glykoproteid gesichert werden. Die von SpRINGer und Stalder (22), Klenk und Uhlendruck $(4,7,22$, 23), Winzler und Kathan (10) mitgeteilte Beeinflussung der MN- und Virus-Rezeptor-Eigenschaften durch enzymatische Neuraminsäure-Abspaltung fanden wir ebenfalls in unseren Untersuchungen. Gegenüber KLENK (7), Uhlendruck $(7,23)$ und Baranowski und Mitarbeitern (20) konnten wir anstelle von 14 Aminosäuren entsprechend den Befunden von KathaN und WINZLER (10) 16 Aminosäuren nachweisen, von denen Serin am stärksten vertreten war. Der wichtigste Befund dieser Untersuchungsergebnisse ist jedoch die Isolierung eines Xylose-haltigen Glykoproteids aus dem ErythrocytenStroma. Dieses erstmalig beschriebene Glykoproteid unterscheidet sich chemisch, serologisch und in dem Virus-Hämagglutinations-Test von dem Glykoproteid (W) mit MN-Antigen-Eigenschaften. $\mathrm{Da}$ es aus der Phenolphase mit der Stroma-Präparation nach WESTPHAL (25) und UHLENBRUCK (15) isoliert wurde, in der auch die AB- Eigenschaften lokalisiert sind, in der jedoch keine MN-Antigen-haltigen Glykoproteide vorkommen, ist es auch in seiner Erythrocyten-MembranLokalisation von dem Glykoproteid (W) zu unterscheiden. Das Glykoproteid (P) konnte erst nach Extraktion der Lipide und Glykolipide freigesetzt werden. $\mathrm{Da}$ der Extraktionsrückstand, aus dem wir diese Substanz isolierten, bis jetzt noch nicht aufgearbeitet wurde, erscheint es nicht erstaunlich, daß das Glykoproteid (P) noch nicht beschrieben worden war. Bei einem XyloseGehalt von 3,0-3,5\% und dem gleichzeitigen Vorliegen von Galaktose, Mannose, Fucose, Galaktosamin, Glucosamin und einer nur partiell abspaltbaren Neuraminsäure ist. es schon aufgrund seiner chemischen Eigenschaften eine untersuchenswerte Substanz. $\mathrm{Da}$ eine Xylose-Konzentration in der Höhe von 3-3,5\% bei Glykoproteiden des menschlichen Organismus bis jetzt noch nicht beschrieben wurde, ist eine bei alkalischer Hydrolyse labile Kohlenhydrat-Peptid-Bindung über Xylose-Serin zu diskutieren. Die Xylose-Serin-Verbindung, die bei den Mucopolysacchariden Heparin und 
Chondroitinsulfat von LINDAHL und RoDÈN (40, 41) bewiesen wurde, könnte auch für das Glykoproteid (P) in Frage kommen, da einmal ein hoher Xylose-Gehalt gefunden wurde und Serin die Aminosäure war, die die höchste Konzentration aufwies. $\mathrm{Da}$ das Glykoproteid $(P)$ eine niedrigere Neuraminsäure-Konzentration besitzt als das Glykoproteid (W), keine MN-AntigenEigenschaften hat, nur geringe Virus-Rezeptor-Fähigkeiten besitzt und die Neuraminsäure nur partiell enzymatisch abspaltbar ist, unterscheidet es sich chemisch und funktionell von dem bis jetzt beschriebenen $\mathrm{MN}$ Antigen-haltigen Glykoproteid (W).

Herrn Dr. D. Roelcke, Serologisches Institut der Universität Heidelberg, danke ich für die Durchführung der serologischen Untersuchungen und Herrn Dr. A. KrUGE, Serologischẹ Institut der Universität Heidelberg, für die großzügige Zurverfügungstellung der notwendigen Blutkonserven. Fräulein G. JENSSEN, Medizinische Universitäts-Poliklinik Heidelberg, danke ich für ibre wertvolle Mithilfe bei allen biochemischen Untersuchungen.

\section{Literatur}

1. Howe, C., J. Immunol., Baltimore 66,9 (1951). - 2. HoHorsT, H.-J., Zschr. Hyg. Berlin 139, 561 (1954). - 3. LisowskA, E., Archwm. Imm. Terap. dóswiad. 8, 235 (1960). - 4. KLENK, E. und H. Lempridi, Hoppe-Seyler's Z. physiol. Chem. 307, 278 (1957). - 5. Klenk, E., H. FaIllard und H. Lemprrid, HoppeSeyler's Z. physiol. Chem. 301,235 (1955). - 6. Morgan, W.T. J., Naturwissenschaften 46, 183 (1959). - 7. KLENK, E. und G. UhlenbruCK, Hoppe-Seyler's Z. physiol. Chem. 319, 151 (1960). 8. UHLENBRUCK, G., Zschr. Immunit.forsch., Jena, 121, 420 (1961). - 9. Eylar, E. W., M. A. Madoff, O. V. Brody und J. L. ONCley, J. biol. Chemistry 237, 1992 (1962). - 10. Kathan, R. H. und R. J. WinzLeR, J. biol. Chemistry 238, 21 (1963). 11. Kathan, R. H., R. J. WinzleR und C. A. Johnson, J. Exper. Med., New York 113, 37 (1960). - 12. UhLEN̄Bruck, G., Vox Sang. 9, 377 (1964). - 13. Cook, G. M. W. und E. H. EyLAR, Biochim. biophysica Acta (Amsterdam) 101, 57 (1963). - 14. Gramlich, F., Die Rezeptorfunktion der Erythrocyten. S. 3, S. Karger Basel-New York (1966). - 15. Uhlendruck, G. und D. O. Schurid, Zschr. Immunit.forsch., Jena 123, 466 (1962). - 16. Kabat, E. A., Blood group substances. Academic Press Inc., New York (1956). - 17. Mäkelä, O. und K. Cantell, Ann. med. exper. biol. Fenniae, 36, 366 (1958). - 18. SpRINGER, G. F. und N. J. Ansell Hahn, Proc. Nat. Acad. Sci., USA, 44, 182 (1958). 19. Romanowska, E., Archwm. Immun. Terap. dóswiad. 7, 759 (1959). - 20. Baranowski, T., E. Lrsowska, A. Morawiecki, E. Romanowska und K. Strozecka, Archwm. Immun. Terap. dóswiad. 7, 15 (1959). - 21. NAgAI, Y. und G. F. SPRINGER,
Federation Proc. 21, 67 (1962). - 22. Sralder, K. und G. F. SpRINGer, Proc. 8th Congr. Europ. Soc. Haematol. Wien 1961, Karger, Basel-New York (1962). - 23. PROKOP, O. und G. UHLENBRUCK, Lehrbuch der menschlichen Blut- und Serumgruppen, Edition Leipzig (1963). - 24. Bishop, Ch. und D. M. Surgenor. The red blood cell. Acad. Press New York-London (1964). - 25. Westphat, O., O. Lüderitz und F. Bister, Z. Naturforsch. 7b, 148 (1952). - 26. YEMM, E. W. und A. J. WrLLIs, Biochem. J. 57, 508 (1954). - 27. WinzLeR, R. J. in D. Grick, Methods of Biochemical Analysis, Bd. 2, S. 279, Intersience Publ. Inc., New York (1955). - 28. Drsche, Z. und L. B. Shetriles, J. biol. Chemistry 175, 595 (1948). - 29. SVENNERHOLM, L., Biochim. biophysica Acta (Amsterdam) 24, 604 (1957). - 30. WARreN, L., J. biol. Chemistry 234, 1971 (1959). - 31. Trschler, I. und D. Peters, Zbl. Bakt.-Abt. I Orig. 195, 408 (1965). - 32. Kuhn, D., D. GräsșLin und H. WeICKER, Klin. Wschr. 45, 1029 (1967). 33. Gregory, J. D., Arch. Biochem. Biophysics 89, 157 (1960). 34. RoE, J. H. und E. W. Rice, J. biol. Chemistry 173, 507 (1948). - 35. Cessi, C. und F. PiLIego, Biochem. J. 77, 508 (1960). - 36. Voмнор, D. W. und T. C. Tucker, J. Chromatogr. 17, 300 (1965). -37. Wallenfels, K., E. Bernt und G. Limberg, Angew. Chem. 23, 581 (1953). - 38. Fischer, F. G. und H. J. NebeL, HoppeSeyler's Z. physiol. Chem. 302, 10 (1955). - 39. GARDELL, S., F. HeijkenskjöLd und A. Rọch-Norlund, Acta chem. scand. 4, 970 (1950). - 40. LINDAHL, U. und L. Rodèn, J. biol. Chemistry 240, 2821 (1965). - 41. LiNDAHL, U. und L. RoDÈN, J. biol. Chemistry 241, 2113 (1966).
Prof. Dr. H. Weicker 69 Heidelberg Hospitalstr. 3 